professional bodies. Clin Med 2006;6:13-5.

6 Saunders J. Institutional ethics committees: lessons from the Royal College of

Physicians? Clin Ethics 2008;3:46-9.

\section{Harveian Oration 2008}

Editor - Sir Michael Rawlins' fascinating review of decision making in the use or approval of therapeutic interventions (Clin Med December 2008 pp 579-88) mentions the belief that the results of the GREAT trial of GP home thrombolysis were 'too good to be true'. Oddly, suspicions of biological implausibity are employed most often by statisticians, rather than doctors, to discount statistical hypotheses. Here, a delay of one or two hours immediately after an infarct could easily halve the benefit of thrombolysis, as was in fact observed in the study. To explain this away, an entirely imaginary prior scenario was introduced to 'pull back' the results to a more acceptable range. This example discredits rather than supports the use of Bayesian analysis. It reminds one of the bad old days when 'We set out to prove (or disprove)...' was tolerated as a preamble to a paper.

GH HALL

Retired Physician, Exeter

\section{In response}

The phrases 'pulled back' and 'too good to be true' were deliberately put in quotes and taken from the reference. They are therefore Stuart Pocock's and David Spiegelhalter's own words. David Spiegelhalter, the doyen of Bayesian statistics, has often used this himself, as an example of the 'appropriate' use of Bayesian statistics. Hence my use in the Oration!

MICHAEL RAWLINS

Chair, National Institute for Health and Clinical Excellence
Having practised as a clinical haematologist for 30 years, I realised that getting the partnership between myself and my patients right was by far the most important priority in my professional life. If the doctor is really at pains to see that the partnership is working, then everything else falls into place, and the patient receives the best care available. If the partnership is working, the doctor will see to it that their colleagues at every level are people the patients feel they can trust, and the patients are usually grateful whatever the clinical outcome. Furthermore, when mistakes are made, as unfortunately they are even in the best-run institutions, the patient is far more likely to be accepting and understanding than litigious and belligerent. The fact that nowadays patients are so much better informed enhances, rather than detracts from, the partnership.

I was fortunate to work in a specialty where many of the patients had medically (as opposed to surgically) the most treatable malignancies, but the above considerations apply equally to patients with nonmalignant diseases. The patient-doctor partnership is of infinitely greater importance than, for example, the type of building we practise in, new or old, polyclinic or hospital. Dame Margaret's 'solution' in her final paragraph is entirely correct - the sooner changes can be brought about to achieve this, the better. If these issues were addressed, morale would improve automatically and many of the current problems would be solved. TONY ROQUES Retired Consultant Haematologist Worthing Hospital

\title{
The patient-doctor partnership
}

Editor - It was very interesting to read Dame Margaret Turner-Warwick's excellent paper (Clin Med December 2008 pp 573-5). She is absolutely right that the patientdoctor partnership 'should be the cornerstone in any healthcare service'.

\section{Clinical es Scientific letters}

Letters not directly related to articles published in Clinical Medicine and presenting unpublished original data should be submitted for publication in this section. Clinical and scientific letters should not exceed 500 words and may include one table and up to five references.

The use of aspirin and dipyridamole in the treatment of acute ischaemic stroke/transient ischaemic attack: an audit-based discussion

The National Institute for Health and Clinical Excellence (NICE) recommends combination therapy (low dose aspirin plus modified-release dipyridamole) for all ischaemic strokes and transient ischaemic attacks (TIAs), for secondary prevention. Combination therapy is recommended for two years and thereafter low dose aspirin alone. $^{1}$

The first such audit at Withybush General Hospital in Haverfordwest was carried out in 2007 and was published as a clinical letter in this journal. ${ }^{2}$ The practice has since been re-audited.

In the re-audit, 108 inpatients were included and data were collected on a proforma.

Eighty-nine patients (82\%) presented with a first episode and 19 (18\%) with recurrence. Of the 89 patients, 47 (53\%) were prescribed the combination therapy and $20(22.5 \%)$ were given aspirin only. Twelve were on warfarin, three on aspirin + warfarin, four on aspirin + clopidogrel and two on clopidogrel only. In the 2007 audit, $12 \%$ had combination therapy and $71 \%$ aspirin alone.

In the recurrent disease group, seven (37\%) were on combination therapy compared to $16(62 \%)$ in 2007 while four $(21 \%)$ were on aspirin alone. Six patients were on warfarin, one on aspirin + warfarin and one on aspirin + clopidogrel.

Only three out of 54 patients on combi- 